EVS28

KINTEX, Korea, May 3-6, 2015

\title{
Design of a Novel SiC MOSFET Structure for EV Inverter Efficiency Improvement
}

\author{
Young-Kyun Jung ${ }^{1}$, Jong-Seok Lee, Taewon Lim \\ Research \& Development Division Hyundai MotorsSeoul, Korea \\ cozykyun@hyundai.com
}

\begin{abstract}
Inverters for electric vehicle motor drive systems are essential in converting the battery's direct current into alternating current. $\mathrm{Si}$ (Silicon) IGBT that is commonly used in inverter modules have large Vce,sat and turn-off time due to $\mathrm{p}+$ drain and tail current. Therefore, inverter modules consist of Si IGBT with relatively low efficiency. If we can use MOSFETs instead of IGBT in inverter modules, it is possible to achieve high efficiency because of short turn-off time and high operating frequency. Yet also has a problem; Si MOSFETs has large on-resistance compared to Si IGBTs. In this study, SiC(Silicon Carbide) was used to make MOSFETs instead of Si. Futhermore, an accumulation channel concept is adapted to a $\mathrm{SiC}$ trench MOSFET, namely Trench ACCUFET. Compared with conventional SiC trench MOSFETs, the novel $\mathrm{SiC}$ trench ACCUFET structure has not only lower on-resistance but also high breakdown voltage as shown by the simulation results. We fabricated the Trench ACCUFET for verification, and described improvements that is to be made.
\end{abstract}

Keywords: SiC, MOSFET, EV, Inverter, Trench

\section{Introduction}

EVs(Electric Vehicles) must be available to travel long distances with the same battery capacity in terms of battery cell efficiency. Thus, the inverter used to drive the motor of EVs should have good power conversion efficiency. In addition, EV inverters must ensure high reliability in harsh automotive environments, such as high temperatures. In order to accomplish this sole purpose, silicon carbide with excellent heat characteristics and low resistance must to be used to design a device instead of silicon. We proposed a new structure of a $\mathrm{SiC}$ trench MOSFET(or trench ACCUFET) with verified simulation results. As a result, breakdown voltage increased by $20 \%$ and on-resistance decreased by $23 \%$, compared with a conventional $\mathrm{SiC}$ trench MOSFETs.

Also we fabricated a trench ACCUFET in order to verify the results and effects. Yet due to some problems with the device fabrication process, we could not confirm the merits of our trench ACCUFET. However, we confirmed the feasibility of the trench ACCUFET concept.

Fig. 1 Shows the structure of the inverter module for driving a 3-phase motor. In order to drive a motor, the inverter system has to convert direct current into alternating current. Inverter module for the operation of the $\mathrm{DC}$ to $\mathrm{AC}$ conversion consists of a total six IGBTs

Table 1 is an example of specifications of the electric vehicle inverter. In comparison to the industrial inverter, the specifications show implicit features and performance for electric vehicle 
inverters to be provided. The output range of inverters, from $50 \mathrm{~kW}$ to $100 \mathrm{~kW}$, covers the range from compact cars to SUVs. Inverter driving power is a DC $240 \sim 400 \mathrm{~V}$ which is considered the battery SOC(State Of Charge) like lithium ion. 12V Lead-Acid batteries are used as a control power like internal combustion engine cars. In Electric Power Train of the EV, the driving force utilizes a motor instead of an internal combustion engine.

However the torque of the motor is continuous in accordance with the speeds, which is different from the internal combustion engine, and, therefore, EV needs a fixed reduction gear ratio instead of multi-stage transmission. Considering the torque characteristics, the speed required for the vehicle, and the deceleration characteristics of $\mathrm{EV}$, it is necessary to control the high-speed of the motor until 12,000 rpm. This range is 4 times more than that of the industrial usage. Also EV inverters require high power density because of limited space and high fuel efficiency.

Therefore, In order to guarantee high reliability, $\mathrm{SiC}$ material having excellent thermal characteristics must be used. Also, in order to ensure high fuel efficiency, power devices having low on-resistance must be designed for high power conversion efficiency.

In this paper, we proposed a $\mathrm{SiC}$ trench ACCUFET structure that has lower on-resistance and higher breakdown voltage compared to conventional $\mathrm{SiC}$ trench MOSFETs. To prove the advantages of our $\mathrm{SiC}$ trench ACCUFET, a prototype was manufactured at the Fraunhofer Institute, Germany.

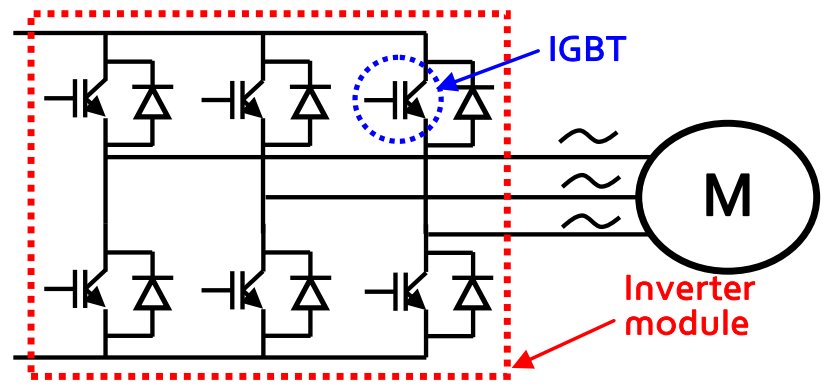

Figure $1: 3$-phase inverter module for motor drive

\begin{tabular}{|c|c|c|}
\hline \multirow{2}{*}{$\begin{array}{c}\text { Output } \\
\text { Power } \\
\text { Rating }\end{array}$} & $\begin{array}{c}\text { Output } \\
\text { power[kW] }\end{array}$ & $50 \mathrm{~kW} \sim 100 \mathrm{~kW}$ \\
\cline { 2 - 3 } $\begin{array}{c}\text { Overload } \\
\text { capacity }\end{array}$ & $200 \% / 2 \mathrm{~min}$ \\
\hline $\begin{array}{c}\text { Input Power } \\
\text { Rating }\end{array}$ & Input power & Battery \\
\cline { 2 - 3 } & Input voltage & DC $240 \sim 400 \mathrm{~V}$ \\
\hline \multicolumn{2}{|c|}{ Control power } & Battery DC $12 \mathrm{~V}$ \\
\hline Enclosure protection conditions & More than IP 65 \\
\hline \multicolumn{2}{|c|}{ Operating speed } & $\sim 12000 \mathrm{rpm}$ \\
\hline \multicolumn{2}{|c|}{ Cooling system } & Forced water cooling \\
\hline \multicolumn{2}{|c|}{ Cooling conditions } & $8 \mathrm{LPM}, 65^{\circ} \mathrm{C}$ \\
\hline \multicolumn{2}{|c|}{ Operating temperature } & $-40^{\circ} \mathrm{C} \sim+85^{\circ} \mathrm{C}$ \\
\hline \multicolumn{2}{|c|}{ Vibration conditions } & $\sim 5 \mathrm{~g}$ \\
\hline EMC & Discrete/vehicle meets \\
\hline \multicolumn{2}{|c|}{}
\end{tabular}

Table 1. An example of inverter specifications for electric cars

\section{DEVICE SIMULATION}

\subsection{Inversion channel trench MOSFET}

Fig. 2(a) shows a cross-sectional view of the inversion channel trench MOSFET structure. The most important feature of this device is the channel between the source and drain p-base region adjacent to the gate oxide, in which an inversion layer(n) is formed by inducing gate voltage. Channel resistance of this device accounts for most of the total resistance because the trench etching of $\mathrm{SiC}$ material is very difficult and the trench side interface state is very poor. It is imperative to decrease the channel resistance to decrease the total on-resistance of $\mathrm{SiC}$ devices.

\subsection{Proposed Accumulation channel trench MOSFET}

Fig. 2(b) shows a cross-sectional view of the proposed accumulation channel trench MOSFET structure. N-type region is formed between the gate oxide and the P-base. Doping concentration and width of the n-type region has to be carefully selected to be depleted completely by the internal potential of the $\mathrm{p}-\mathrm{n}$ junction when no voltage is applied to the MOS gate. Thus, operates as a normally-off device. When positive gate bias is applied, accumulation channel is spread widely from the $\mathrm{SiO} 2 / \mathrm{SiC}$ interface to the p-base. ${ }^{1)-4)} \mathrm{Fig}$. 3 shows the flow of electron current. In case of an accumulation channel trench MOSFET, the width of the channel formed by the same gate voltage is much wider. ${ }^{5)}$ Consequently, the channel resistance of an accumulation channel MOSFET is lower than that of an inversion channel MOSFETs 
by more than twofold. This is the key point of the accumulation channel concept, which indicates a decreasing total on-resistance of the device.

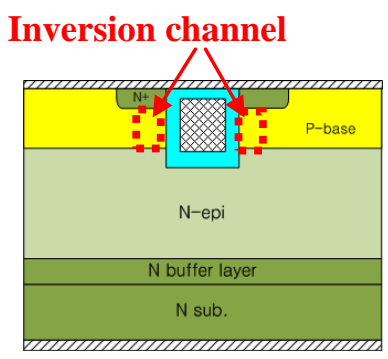

(a)

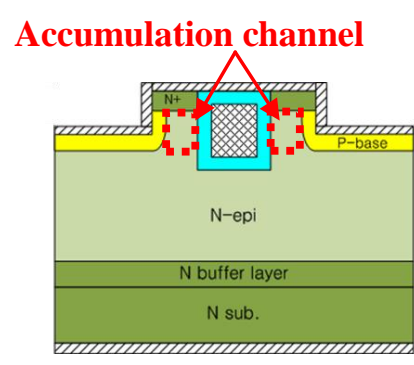

(b)
Figure 2. Trench gate MOSFET structure of (a) previous inversion channel, and (b) proposed accumulation channel

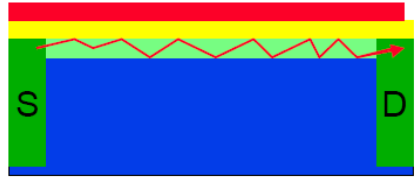

(a)

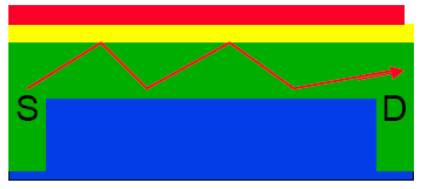

(b)
Figure 3. Current flow of (a) inversion channel and (b) accumulation channel.

Fig. 2(a) shows a cross-sectional view of the inversion channel trench MOSFET structure. The most important feature of this device is the channel between the source and drain p-base region adjacent to the gate oxide, in which an inversion layer(n) is formed by inducing gate voltage. Channel resistance of this device accounts for most of the total resistance because the trench etching of $\mathrm{SiC}$ material is very difficult and the trench side interface state is very poor. It is imperative to decrease the channel resistance to decrease the total on-resistance of $\mathrm{SiC}$ devices.

\subsection{Simulation results}

To compare the simulation results, the same design parameters are applied to the conventional trench MOSFET and the proposed trench ACCUFET as follows:

The doping concentration of $\mathrm{N}$ substrate is $5 X 1018 \mathrm{~cm}-3$, n buffer layer $5 X 1018 \mathrm{~cm}-3$, n- epi layer $5 \times 1015 \mathrm{~cm}-3, \mathrm{p}$ base $1 \times 1017 \mathrm{~cm}-3, \mathrm{n}+$ source layer $1 \mathrm{X} 1018 \mathrm{~cm}-3$, and n-epi thickness $7 \mu \mathrm{m}, \mathrm{p}$ base $2.5 \mu \mathrm{m}, \mathrm{n}+$ source layer $0.5 \mu \mathrm{m}$. The depth of the trench gate is $3 \mathrm{\mu m}$. As shown in Fig. 4 , the breakdown voltage of the inversion channel trench MOSFET is $676 \mathrm{~V}$. However, the breakdown voltage of the proposed trench ACCUFET is $813 \mathrm{~V}$ with an inversion channel trench MOSFET, which indicates an increase of roughly $20 \%$. As shown in Fig. 5, the electric field distribution of the trench gate bottom is plotted when applying the same voltage $(676 \mathrm{~V})$ between drain and source. The electric field of the trench ACCUFET is lower than that of the conventional trench MOSFET at gate bottom region ( $\mathrm{x}=8 \sim$ $9.05 \mu \mathrm{m})$. This is because the trench ACCUFET has $\mathrm{p}$ curved portion( $\mathrm{p}$ base) in the trench gate sides; the $\mathrm{p}$ base divide the electric field concentrated at the bottom of the trench gate oxide. In order to simulate the on-resistance, current-voltage characteristic curves are compared with both devices. On-resistance of the conventional trench MOSFET is $5.62 \mathrm{~m} \Omega \cdot \mathrm{cm}^{2}$ and that of the proposed trench ACCUFET is $4.19 \mathrm{~m} \Omega \cdot \mathrm{cm}^{2}$, which indicates a reduction of on-resistance by approximately $23 \%$. This is the reason behind total resistance reduction, which is comparatively affected by the resistance of the channel region.

The electron current in conventional trench MOSFETs flow through the inversion channel formed at $\mathrm{p}$ base, which is adjacent to the gate oxide. Yet, the electron current in the proposed trench ACCUFET flow much more through the accumulation channel formed at the existing nregion. The accumulation channel makes the electron current flow more deeply from $\mathrm{SiO} 2 / \mathrm{SiC}$ interface by same gate voltage than the inversion channel. The electrons can be moved easily at the accumulation channel with less influence of the oxide interface charge.6) Therefore, it is possible to reduce the large channel resistance of the device having a trench gate. As a result, electron current flows from the source to the drain with a lower resistance compared to inversion channel trench MOSFETs.

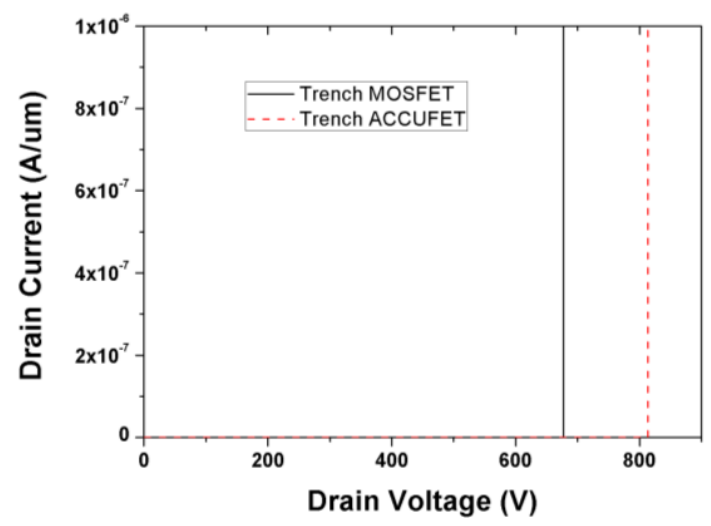

Figure 4. Simulation results of breakdown voltage 


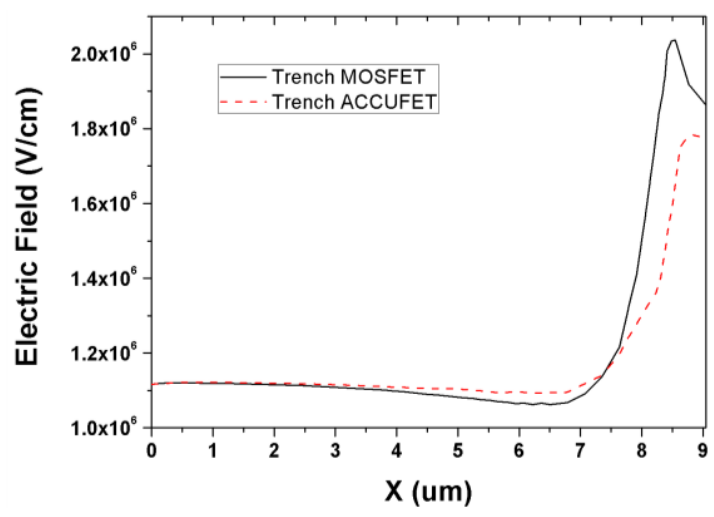

Figure 5. Simulation results of electric field at trench gate bottom

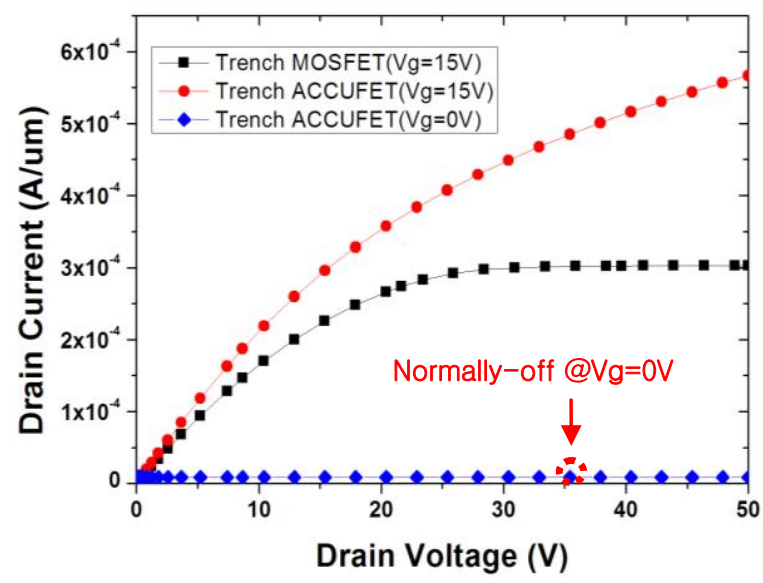

Figure 6. Simulation result of forward I-V characteristics

\section{DEVICE FABRICATION}

\subsection{Process flow}

A brief description of the fabrication process is as follows: (a) n-epi layer formation, and trench etching to form the $\mathrm{p}$ base region; (b) $\mathrm{n}$ type ion implantation to form a source region; (c) gate oxide deposition and poly-Si gate electrode deposits after etching the trench gate region.

The key is to to adjust the spacing (ps) within sub-micron levels between the trench gate and the $\mathrm{p}$ base. If the spacing(ps) is more than submicron, it is impossible to operate as a normallyoff switch.

This can be accomplished fully with the current alignment process. The most important feature of this structure is that the electron mobility can be improved by the n-epi accumulation channel and the electron mobility do not be affected by oxide interface charges. Another important feature is that it is not necessary to go beyond $2.5 \mu \mathrm{m}$ depth of ion implantation for $\mathrm{p}$-base region. This is because such effects can be obtained by etching the source trench up to $2 \mu \mathrm{m}$ and implanting p-type only $0.5 \mu \mathrm{m}$. In fact, the current existing equipment cannot process ion implantation of $\mathrm{SiC}$ material equal to or greater than depth of $1 \mu \mathrm{m}$.

\subsection{Fabricated device results}

Fig. 7. shows the fabricated $\mathrm{SiC}$ trench ACCUFET and cross-section SEM images. The experiment results show that it was possible to obtain a desired shape of trench source and trench gate. Compared to the simulation results, the measurement results of the manufactured device did not match, yet the gate leakage current showed a close similarity, as shown in Fig. 8. Although the normally-off characteristics could not be verified from the fabricated devices, a current change in respect to gate voltage was confirmed, as shown in Fig. 9.

Enhancement of device fabrication is needed for the following reasons:

(1) Low implantation energy(Normally on) - Since the $\mathrm{Al}(\mathrm{p}$ type) ion implantation energy was low, it was not possible to obtain the sufficient depth of trench source $\mathrm{p}$-base $(\mathrm{Tp}$-base $=\min$. $0.5 \mathrm{um}$ needed) to make a fully-depleted region between gate oxide and trench p-base(Fig. 10). Although the gate voltage was zero, the currents flowed from the drain to the source metal contact.

(2) Gate oxide etch mask mis-align(Low drain current) - Since FOX on $\mathrm{n}+$ source was not completely eliminated, it was not possible to contact source metal with one side $n+$ source. As a result, channel density was decreased to about half compared with the simulation results(Fig. 11). Additionally, sufficient accumulation channel was not formed by the gate voltage due to thick gate oxide.
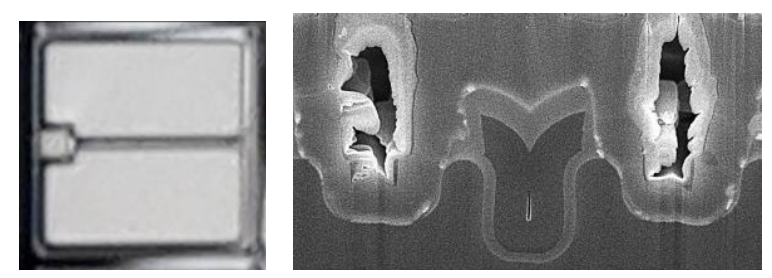

Figure 7. Fabricated $\mathrm{SiC}$ trench ACCUFET and cross section $(\mathrm{SEM})$ - source trench and gate trench are shown 

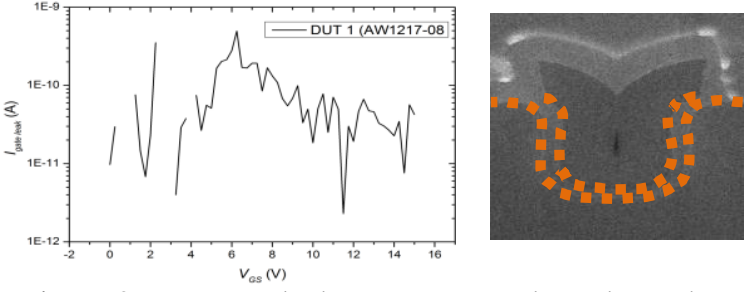

Figure 8. Low gate leakage current and good trench gate shape $(<1 \mathrm{nA} @ \mathrm{VGS}=20 \mathrm{~V}, \mathrm{VDS}=0 \mathrm{~V})$

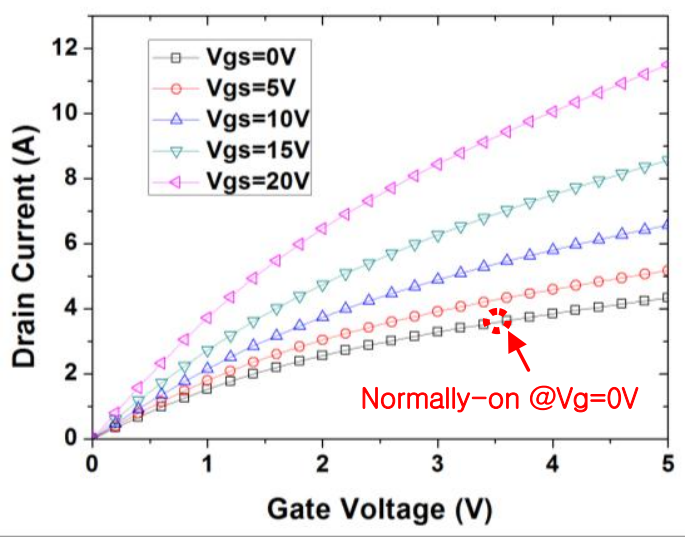

Figure 9. Forward I-V characteristics

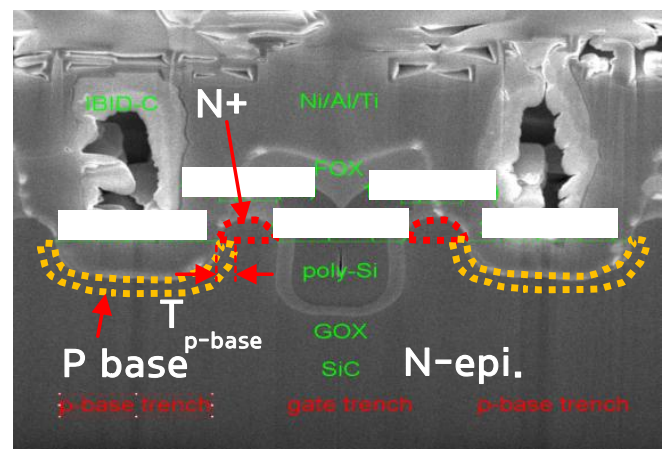

Figure 10. Cross-section of the device shows a shallow p-base depth(Tp-base)

- not fully depleted channel region

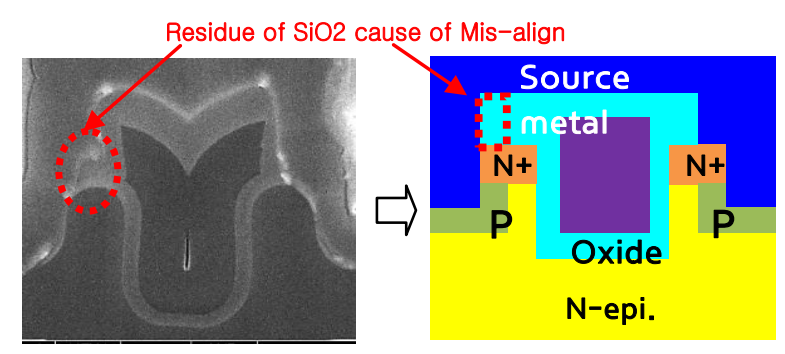

Figure 11. Trench gate FOX residues in SEM and outline illustration

\section{CONCLUSION}

If such drawbacks are solved, it can be concluded that a novel $\mathrm{SiC}$ trench ACCUFET operating in the normally-off mode can be made, with sufficiently high current. The effects of the new MOSFET structure proposed in this paper are organized as follows:

(1) The resistance is greatly reduced. One of the whole device channel resistance is greatly reduced in order to use the accumulation channel than the conventional inversion channel of trench MOSFETs.

(2) The electric fields concentrated below the trench gate bottom are dispersed in p-base/n-epi junction. Thus the breakdown voltage of the device increases dramatically.

(3) Through a relatively simple process, the breakdown voltage and the total on-resistance are both improved.

Finally, our trench ACCUFET device with modified process is under fabrication in order to solve such drawbacks. The device will have a lower on-resistance than conventional trench MOSFETs and is expected to show normally-off characteristics.

\section{References}

[1] Ranbir Singh, Member, IEEE, D. Craig Capell, Mrinal K. Das, Lori A. Lipkin, and John W. Palmour, Member, IEEE, "Development of HighCurrent 4H-SiC ACCUFET", IEEE Trans. on Electron Devices, Vol. 50, No. 2, Feb. 2003.

[2] Ravi K. Chilukuri, Praveen M. Shenoy, and B. Jayant Baliga, Fellow, IEEE, "High-Temperature Operation of SiC Planar ACCUFET", IEEE Trans. on Industry Applications, Vol. 35, NO. 6, NOVEMBER/ DECEMBER 1999.

[3] Tesfaye Ayalew, Jong-Mun Park, Andreas Gehring, Tibor Grasser, and Siegfried Selberherr, "Silicon Carbide Accumulation -Mode Laterally Diffused MOSFET", IEEE, 2003.

[4] Praveen M. Shenoy and B. Jayant Baliga, "The Planar 6H-SiC ACCUFET: A New High-Voltage Power MOSFET Structure", IEEE ELECTRON DEVICE LETTERS, VOL. 18, NO. 12, DECEMBER 1997.

[5] Fathipour, M., Peyvast, N. and Shoaazar, N., "A Novel 6H-SiC UMOSFET ACCUFET with Low Specific On-resistance and Peak Electric Field", Optoelectronic and Microelectronic Materials and Devices, 2008.

[6] Liewih, H., Dimitrijev, S., Weitzel, C. E. and Harrison, H. Barry, "Novel SiC accumulation-mode power MOSFET", IEEE Transactions on Electron Devices, Vol. 48, Issue 8, 2001. 